\title{
A (in)formação científica e humanizada dos profissionais da área de saúde: a literatura nas humanidades médicas
}

\author{
The scientific and humanized information for training of health \\ professionals: literature in the medical humanities
}

\section{La (in)formación científica y humanizada de los profesionales de la salud: la literatura en las humanidades médicas}

\author{
Patrícia de Almeida $a^{1, a}$ \\ mebpatricia@gmail.com | http://orcid.org/0000-0002-8061-8622 \\ ${ }^{1}$ Universidade de Coimbra, Faculdade de Letras. Coimbra, Portugal. \\ a Especialização Avançada em Ciência da Informação pela Universidade de Coimbra.
}

\section{Resumo}

Literatura e medicina convivem harmoniosamente, sendo trivial afirmar que ler faz bem à saúde. Todavia, o diálogo entre literatura e ciência nem sempre é assumido em benefício mútuo. A literatura faz bem à medicina e mostra-se útil à (in)formação dos profissionais de saúde? Com o objetivo de refletir sobre este assunto, efetuou-se uma auscultação da literatura científica recente sobre essa temática. Como resultado, enumeraram-se os trabalhos considerados mais relevantes, com contributos concretos da literatura para a medicina e das humanidades para a educação em ciências médicas. Conclui-se que os profissionais de saúde valorizam a literatura na prática clínica e que a neurologia e a psiquiatria são as áreas que mais reconhecem o potencial literário. Reconhece-se uma tendência à inclusão das humanidades médicas nos currículos dos cursos das ciências da saúde. Assim, será benéfica a inclusão da literatura nas humanidades médicas e na formação dos profissionais de saúde.

Palavras-chave: Humanidades médicas; Literatura; Formação médica; Ciências da saúde; Informação científica.

\begin{abstract}
Literature and medicine coexist harmoniously, being trivial to say that reading is good for health. However, the dialogue between literature and science is not always assumed for their mutual benefit. Is literature good for medicine and useful for training of health professionals? Aiming to reflect on this subject, a study of recent scientific literature on this subject was carried out. As a result, we have listed the works considered most relevant, with concrete contributions from the literature for the medicine and from the humanities for the education in medical sciences. We concluded that the health professionals value the literature in
\end{abstract}


clinical practice and that the neurology and the psychiatry are the areas that most recognize the literary potential. A trend towards the inclusion of medical humanities in the curricula of health science courses is acknowledged. Thus, it will be beneficial to include literature in the medical humanities and in the training of health professionals.

Keywords: Medical humanities; Literature; Medical education; Health sciences; Scientific information.

\section{Resumen}

La literatura y la medicina conviven armoniosamente, siendo trivial afirmar que leer hace bien a la salud. Sin embargo, el diálogo entre literatura y ciencia no siempre es asumido en beneficio mutuo. ¿La literatura hace bien a la medicina y se muestra útil a la (in)formación de los profesionales de salud? Con el objetivo de reflexionar sobre este asunto, ha se efectuado una auscultación de la literatura científica reciente sobre esta temática. Como resultado, se han enumerado los trabajos considerados más relevantes, con contribuciones concretas de la literatura para la medicina y de las humanidades para la educación en ciencias médicas. Se concluye que los profesionales de salud valoran la literatura en la práctica clínica y que la neurología y la psiquiatría son las áreas que más reconocen el potencial literario. Se reconoce una tendencia a la inclusión de las humanidades médicas en los currículos de los cursos de las ciencias de la salud. Así, será benéfica la inclusión de la literatura en las humanidades médicas y en la formación de los profesionales de la salud.

Palabras clave: Humanidades médicas; Literatura; Formación médica; Ciencias de la salud; Información científica.

Contribuição dos autores: todas as etapas do trabalho foram realizadas por Patrícia de Almeida.

Declaração de conflito de interesses: Este trabalho não apresenta conflito de interesses.

Fontes de financiamento: Sem fontes de financiamento.

Considerações éticas: não há.

Agradecimento/Contribuições adicionais: não há.

Histórico do artigo: Submetido: 18.abr. 2018| Aceito: 01.ago.2018 | Publicado: 25.set.2018

Apresentação anterior: não houve.

Licença CC BY-NC atribuição não comercial. Com essa licença é permitido acessar, baixar (download), copiar, imprimir, compartilhar, reutilizar e distribuir os artigos, desde que para uso não comercial e com a citação da fonte, conferindo os devidos créditos de autoria e menção à Reciis. Nesses casos, nenhuma permissão é necessária por parte dos autores ou dos editores. 


\section{Introdução}

$\mathrm{Na}$ atualidade, a literatura e a medicina convivem harmoniosamente, sendo comum afirmar que ler faz bem à saúde. Isto já foi inclusive reconhecido pela comunidade científica e existe registro de vários projetos de leitura terapêutica em instituições médicas. A título de exemplo, apontam-se o projeto de voluntariado "Ler faz bem" do Hospital de Santo António no Porto - Portugali, onde se promove a leitura semanal em voz alta a utentes e familiares nos serviços de neurologia, nefrologia e neurofisiologia, e o projeto desenvolvido em hospitais da cidade baiana de Itabuna, com efeitos benéficos tanto nos pacientes como nos mediadores de leitura ${ }^{1}$. Portanto, a associação da literatura à saúde já é trivial.

No entanto, tratando-se de medicina, o diálogo com a literatura nem sempre é claramente assumido em benefício mútuo. Regra geral, destacam-se os contributos que a medicina pode trazer à literatura, especialmente como fonte de informação, de inspiração e assunto para obras literárias, e referemse os médicos escritores de ficção. No primeiro caso, lembra-se Fiódor Dostoiévski, um escritor que soube transformar o seu vulgar transtorno neurológico (epilepsia) em matéria literária extraordinária, convertendo a adversidade de uma doença em oportunidade artística ${ }^{2}$. No segundo, recordam-se casos de célebres escritores e médicos, como o russo Anton Checov, o psiquiatra português António Lobo Antunes ou o brasileiro Guimarães Rosa ${ }^{3,4}$, que tão bem souberam materializar o seu saber médico e a sua experiência clínica em narrativas literárias.

Assim, considera-se que a medicina pode potenciar a arte e faz bem à literatura. Contudo, afirmar que a literatura promove e beneficia a medicina suscita algumas interrogações: como poderá a arte beneficiar a ciência? Especificamente, como poderá a literatura beneficiar a medicina? A literatura será útil à (in) formação dos profissionais de saúde? Com o objetivo de refletir sobre este assunto e de responder a essas questões, efetuou-se uma auscultação da literatura científica mais recente (desde 2010) sobre a relação benéfica entre a literatura e a medicina e sobre as humanidades médicas, enumerando-se alguns contributos concretos dos textos artísticos para a prática clínica e apontando-se as atuais tendências na formação em ciências médicas.

\section{A literatura na medicina}

Nos últimos anos, vários investigadores têm produzido pesquisas científicas, cujos resultados mostram que a literatura contribui beneficamente para a medicina. Iniesta ${ }^{2-5}$ considera que a literatura ficcional complementa a literatura médica na formação contínua em neurologia e fala de uma relação de simbiose entre escritores e médicos. Esse neurologista espanhol afirma que Freud, Alajouanine e Gastaut se inspiraram na epilepsia de Dostoiévski para desenvolverem as suas teorias e a ideia de que a literatura oferece uma perspectiva humanizadora dos relatos patológicos. Isto pode acontecer tanto por esclarecer sobre aspectos não abordados pela ciência (por exemplo, a vivência das doenças por pacientes e seus familiares), como por contribuir para erradicar o estigma social associado a algumas enfermidades.

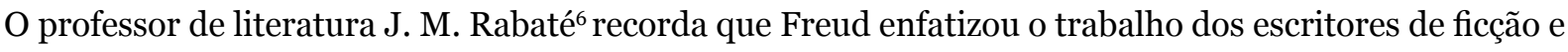
o que eles podem ensinar sobre o inconsciente do ser humano, mesmo sem terem essa intenção pedagógica. Este é o motivo por que Freud designou a literatura como uma componente essencial para a formação de um analista competente. Por sua vez, a médica pneumologista Terezinha Vargas e a psicanalista Vera Pollo ${ }^{7}$ lembram que Freud sempre valorizou o papel da arte na prática clínica, em especial o da poesia, sendo que esta lhe serviu de guia na construção da psicanálise. A doutora em literatura e psicóloga Priscila Marques ${ }^{8}$ também confirma que a "crítica psicológica", isto é, aquela realizada por médicos e por psicólogos, fez apropriações da literatura dostoievskiana.

i Ver https://www.jn.pt/local/videos/interior/voluntarios-leem-historias-e-ajudam-doentes-a-sair-do-hospital-9040908.html 
Em outro trabalho, Iniesta9 ${ }^{9}$ explica a importância da medicina baseada na narrativa em situação de consulta, já anteriormente salientada por neurologistas como Alzheimer ou Oliver Sacks: quanto maior for a discrepância entre a experiência da doença por parte do paciente e a interpretação que dela faz o médico, menor será a probabilidade de a relação entre ambos ser clinicamente frutífera. Osvaldo Barison ${ }^{10}$, membro efetivo da Sociedade Brasileira de Psicanálise de São Paulo, destaca a importância da recriação de afetos possibilitados por uma narrativa, exemplificando com a semelhança entre as sessões de psicanálise e as experiências emocionais sentidas por um leitor.

Em 2015, foi organizado Popularizing dementia: public expressions and representations of forgetfulness, por Aagje Swinnen (professora no Department of Literature and Art na Maastricht University, Holanda) e Mark Schweda (pesquisador no Department of Medical Ethics and the History of Medicine na University of Göttingen, Alemanha) ${ }^{11}$. O livro encontra-se dividido em três partes, sendo logo a primeira designada por Literary fiction. Nela, observa-se como a demência é retratada na literatura contemporânea e conclui-se que a narrativa ficcional permite obter informação sobre essa doença e sobre aqueles que com ela sofrem, seja direta (pacientes) ou indiretamente (familiares e amigos). Salienta-se a análise de dois romances literários, efetuada por Sadie Wearing, nos quais se abordam os impactos da enfermidade, nomeadamente as vulnerabilidades dos doentes e as responsabilidades dos seus cuidadores; essa autora conclui que a literatura pode conter informação relevante sobre questões filosóficas e práticas relacionadas com a doença.

O professor de História da Medicina e doutor em Medicina e Cirurgia Rafael Huertas ${ }^{12}$ analisou os conteúdos psiquiátricos de um romance publicado em 1979, Los renglones torcidos de Dios, de Torcuato Luca de Tena. Em 2017, Huertas avaliou a forma como esse texto ficcional foi capaz de espelhar a realidade assistencial espanhola da época no âmbito da doença mental (loucura), num contexto de profundas transformações culturais. Em vez de conclusões, ele termina a sua investigação com interrogações sobre as limitações da medicina: serão os psiquiatras capazes de diagnosticar e tratar uma mente superior (como a da personagem do romance em causa) com as suas teorias, métodos e protocolos terapêuticos? Podem a ciência e a razão intervir numa situação como a retratada no romance literário?

António Damásio, famoso médico neurologista e professor na Universidade do Sul da Califórnia,

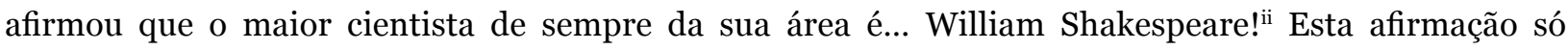
surpreende quem não conhece o trabalho publicado por esse neurocientista português sobre a relação entre o cérebro e as emoções humanas. Em 1994, com O erro de Descartes ${ }^{13}$, ele inovou ao interligar o sistema límbico (parte do cérebro que controla as emoções) e o neocórtex (parte do cérebro que controla a razão), afirmando que ambos trabalham sempre em conjunto. A sua teoria continuou a desenvolver-se ao longo dos anos e, recentemente, em A estranha ordem das coisas ${ }^{14}$, o médico explica e reflete sobre a ação criadora dos sentimentos.

À semelhança do que ocorre em outras áreas científicas, os médicos que escrevem ficção em momentos de ócio transferem-lhe os seus saberes específicos, pelo que se pode afirmar que existe informação científica na ficção literária, através do que se considera um mecanismo de transferência de conhecimento não formal ${ }^{15}$. O psicólogo e enfermeiro de saúde mental Henry Bladon ${ }^{16}$ sugere que os psiquiatras podem contribuir para melhorar problemas da atualidade através da ficção narrativa em que façam refletir o seu conhecimento médico, por exemplo, o estigma associado a algumas doenças.

Nesse sentido, Jonh Launer ${ }^{17}$ fala de Sterben, de Arthur Schnitzler (1862-1931), obra literária que aborda o último ano de vida do protagonista Felix. Muito embora Launer tenha iniciado o seu percurso acadêmico na área da literatura em Cambridge, acabou por se tornar médico de família no Middlesex Hospital de Londres. Ele afirma que a leitura dessa narrativa de Schnitzler não é uma experiência agradável, mas é perfeita para contradizer a ideia de que só se pode aprender sobre a mente, relações, doença, morte e

ii Ver https://www.publico.pt/2017/11/05/ciencia/entrevista/antonio-damasio-1791116 
condição humana através de textos médicos e da ciência, no seu sentido mais tradicional. Este médico afirma mesmo que "I know of no better story to read, in order to understand what medicine can learn from fiction”" ["Não conheço nenhuma história melhor a ser lida para compreender o que a medicina pode aprender com a ficção”].

De uma forma geral, verifica-se que a maioria dos autores aqui citados e que apontam concretos contributos da literatura para a medicina são profissionais de saúde da área das ciências médicas, com destaque para a neurologia e a psiquiatria, como as especialidades que mais reconhecem e legitimam o potencial da literatura para a informação e formação científica dos profissionais de saúde.

\section{As humanidades na formação em ciências médicas}

Os trabalhos recentes da área mostram que as humanidades estão a ganhar terreno na formação em ciências médicas. Esta tendência é confirmada na pesquisa de Sousa, Gallian e Maciel ${ }^{18}$, que fazem uma revisão de literatura nas bases de dados Ovid-SP e Scopus. Esses pesquisadores confirmam que, a partir da década de 1950 do século passado, as designadas "humanidades médicas" têm vindo a crescer como disciplina em escolas médicas, paralelamente nos Estados Unidos da América e no Reino Unido, sendo este país o berço do atual movimento que considera a integralidade como uma necessidade na formação médica. Nesse trabalho, os autores salientam que se referem concretamente à área da literatura como pertencente às humanidades médicas. Muito embora as áreas geográficas de seu estudo, verificam-se movimentos em outros países como, por exemplo, congressos no Uruguai ${ }^{\mathrm{iii}}$, Brasiliv ${ }^{\mathrm{i}}$, Chile ${ }^{\mathrm{v}}$ ou Espanha ${ }^{\mathrm{vi}}$.

Launer ${ }^{16}$ confirma essa tendência e relata que algumas escolas de medicina oferecem cursos de literatura como parte de seu currículo, no âmbito das humanidades médicas, com o intuito de ampliar a compreensão dos alunos sobre as experiências de vida, muito mais bem retratadas nos textos ficcionais do que nos textos científicos.

Cavalcante, Leite, Neves Filho, Sidrim e Silva ${ }^{19}$ reconhecem o destaque que as humanidades médicas vêm ganhando nos currículos, com o intuito de ajudar a compreensão do ser humano, do seu sofrimento e dos seus sentimentos. Na sua pesquisa, por meio de entrevistas com estudantes e profissionais da área médica, concluem que aqueles que tiveram a oportunidade de ter contato com as humanidades médicas proporcionam um atendimento mais consciente e humanizado, o que é benéfico não só para si próprios como também para os pacientes.

No Brasil, Ayres, Rios, Schraiber, Falcão e Mota $^{20}$ relatam o desenvolvimento de uma disciplina no domínio das humanidades médicas, na Faculdade de Medicina da Universidade de São Paulo, abordando quatro áreas: filosofia, história, socioantropologia e psicodinâmica do encontro clínico. De acordo com a investigação realizada, o impacto dessa disciplina foi muito positivo, tanto para os pacientes como para os futuros médicos, e obteve boa avaliação e aceitação de alunos e professores. Salientam-se os ganhos concretos na aquisição de conhecimento relativo ao ponto de vista da integralidade em saúde, razão pela qual os investigadores concluem que a formação dos alunos de graduação médica se beneficia da inserção das humanidades na estrutura curricular. Nesse caso concreto, nota-se a ausência de uma referência direta ao contributo da literatura para a medicina.

Ainda como exemplos brasileiros mais recentes dessa tendência, apontam-se a criação do curso de especialização de Ciência, Arte e Cultura na Saúde ${ }^{21}$, bem como a experiência com alunos do primeiro ano de

\footnotetext{
iii Ver http://www.fnr.gub.uy/curso_humanidades_med_2018.

iv Ver https://portal.cfm.org.br/index.php?option=com content\&view=article\&id=27104:2017-08-10-15-56-11\&catid=3 e http:// www.eventos.cfm.org.br/index.php?option=com content\&view=article\&id=21103.

v Ver http://medicina.uc.cl/noticias/coloquios-humanidades-medicas-la-importancia-de-la-interdisciplinariedad/.

vi Ver http://www.fpablovi.org/index.php/eventos-bioetica/211-vi-simposio-de-humanidades-medicas.
} 
ciências biomédicas da Universidade Federal de São Paulo, onde se discutiu Frankenstein, de Mary Shelley, com o objetivo específico de proporcionar uma visão diferente do ser humano e das relações sociais ${ }^{22}$.

Em Portugal, cabe salientar o caso da Faculdade de Medicina da Universidade do Porto (FMUP) que, em 2014, efetuou uma reforma curricular com valorização de disciplinas na área das humanidades ${ }^{\mathrm{vi}}$, mas na qual não se encontra uma referência específica à literatura. Talvez não tenha sido coincidência que tal reforma tenha ocorrido no ano em que Maria Amélia Ferreira tomou posse como diretora da FMUP. Em entrevista recente ${ }^{\text {viii }}$, ela assume a importância que as humanidades têm na formação médica: "Nós não queremos só técnicos, queremos gente que cuide de nós!” Essa professora da FMUP assume que é fundamental que os futuros médicos saibam gerir informação, pensar criticamente e comunicar-se com os pacientes. Maria Amélia Ferreira compreende o novo cenário educativo que se impõe na formação médica e a necessidade da aquisição de competências transversais (humanísticas e técnicas) para lidar com a fragilidade do ser humano, numa perspetiva integral e humanizadora da prestação de cuidados de saúde ${ }^{23}$.

As humanidades médicas proporcionam um olhar diferenciado que aproxima o profissional de saúde do paciente e constitui com certeza um valor a mais na formação. Neste âmbito, a literatura permitirá uma maior profundidade dos conhecimentos dos futuros profissionais e uma maior consciência da complexidade da realidade e das consequências dos procedimentos médicos. Dessa forma, poder-se-á afirmar que os estudantes de medicina ficam mais bem preparados para cumprir os seus deveres e enfrentar os seus desafios profissionais, e considerar o texto literário também como um recurso pedagógico para a educação das emoções ${ }^{24}$.

Muito recentemente, Gomes, Higa, Passos, Soares, Otani e Souto ${ }^{25}$ realizaram um estudo sobre a perspectiva da integralidade no tratamento do ser humano, isto é, o cuidado centrado na pessoa e no seu contexto familiar e comunitário. Pela ótica dos estudantes de medicina e de enfermagem no domínio da atenção primária à saúde, esses investigadores concluíram que os futuros médicos e enfermeiros aceitam e valorizam o cuidado integral em saúde.

Dessa forma, a tendência da inclusão das humanidades nos currículos dos cursos das ciências da saúde vai-se (con)firmando e o crescimento progressivo desta tendência é a prova da sua necessidade. Como referiu Damásio ${ }^{14}$, é fundamental conciliar a emoção com a razão e nunca como hoje se precisou tanto das humanidades para criar homens e cientistas. A medicina é uma área de natureza dual (ciência e arte), cuja formação deve incluir componentes científicos e humanísticos ${ }^{19}$. Verifica-se, contudo, que o domínio da literatura nem sempre é incluído nas humanidades médicas, uma vez que não há consenso sobre a carga horária curricular e sobre as áreas que delas devem fazer parte ${ }^{26,27}$.

\section{Considerações finais}

Revistos alguns trabalhos acadêmicos sobre a área, considera-se que se poderá responder afirmativamente às questões colocadas. Sim, a arte pode beneficiar a ciência. Sim, a literatura pode beneficiar a medicina. Sim, a literatura será útil à (in)formação dos profissionais de saúde. De forma resumida, verifica-se que as obras literárias inspiram e oferecem uma perspetiva integral e humanizadora à prática clínica; proporcionam empatia com os doentes; fornecem informações para uma melhor compreensão das doenças; e complementam a formação científica dos profissionais de saúde.

Isto é, de fato, assumido por estudantes e por profissionais de saúde, motivo pelo qual está definitivamente aberta uma porta para que literatura e medicina entrem juntas na compreensão propedêutica e terapêutica da integralidade do ser humano. Se a inclusão de uma componente (artística) literária na formação

vii Ver https://sigarra.up.pt/fmup/pt/ucurr_geral.ficha_uc_view?pv_ocorrencia_id=332352.

viii Entrevista disponível em https://www.publico.pt/2017/12/18/sociedade/noticia/do-genero-9-a-primeira-mulher-a-ocupar-ocargo-1796380 
acadêmica especializada dos profissionais de saúde é vista como benéfica, então é hora de assumir que um médico e um enfermeiro são muito mais do que simples técnicos e de incluir a literatura como uma componente essencial das humanidade médicas.

Como se viu, algum caminho já vem sendo feito no sentido de incorporar as humanidades na formação médica, porém muito mais urge ser trabalhado e estudado, nomeadamente no domínio da literatura, para que esta se assuma clara e definitivamente como (in)formação científica nos cursos das ciências da saúde.

\section{Referências}

1. Santos G, Guevara I, Soares K, RibeiroT, Moura W. Ler faz bem à saúde: leitura terapêutica em ambientes hospitalares. Rev PROEX. 2017;5(7):9-18.

2. Iniesta I. La epilepsia en la gestación artística de Dostoievski. Neurología [Internet]. 2014 [citado em 2018 ago 15];29(6):371-8. doi: https://doi.org/10.1016/j.nrl.2011.05.002

3. Lacerda-Queiroz N, Queiroz Sobrinho A, Teixeira AL. As representações da malária na obra de João Guimarães Rosa. Hist cienc saude Manguinhos [Internet]. 2012 [citado em 2018 ago 15];19(2):475-89. doi: http://dx.doi.org/10.1590/S0104-59702012000200007

4. Goulart E. O viés médico na literatura de Guimarães Rosa. Belo Horizonte: UFMG; 2011.

5. Iniesta I. Neurología y literatura. Neurologia [Internet]. 2010 [citado em 2018 ago 15];25(8):507-14. doi: https://doi.org/10.1016/j.nrl.2010.07.013

6. Rabaté JM. Psicanálise e literatura: Por que, hoje? Trivium [Internet]. 2017 [citado em 2018 ago 15];9(2):162-71. doi: http://dx.doi.org/10.18379/2176-4891.2017v2p.162

7. Vargas T, Pollo V. "Ifigênia em Áulis": a guerra, as núpcias e a morte (Contribuições psicanalíticas para uma leitura da tragédia). Trivium [Internet]. 2017 [citado em 2018 ago 15];9(2): 232-45. doi: http:// dx.doi.org/10.18379/2176-4891.2017v2p.232

8. Marques P. Dostoiévski e a Psicologia: o escritor como leitor e objeto da ciência da mente. Cadernos de Literatura Comparada [Internet]. 2017 [citado em 2018 ago 15];37:147-62. doi: http:/dx.doi. org/10.21747/21832242/litcomp37a7

9. Iniesta I. Neurología y Literatura 2. Neurologia [Internet]. 2014 [citado em 2018 ago 15];29(4):242-8. doi: https://doi.org/10.1016/j.nrl.2011.02.007

10. Barison O. A escuta da escrita da escuta: emoções através do relato clínico. J psicanal [Internet]. 2017 [citado em 2018 ago 15];50(93):225-34. Disponível em: http://pepsic.bvsalud.org/pdf/jp/v50n93/ v50n93a17.pdf

11. Swinnen A, Schweda M, editors. Popularizing dementia: public expressions and representations of forgetfulness Bielefeld: Transcript-Verlag; 2015. (Aging Studies, n. 6).

12. Huertas R. Psiquiatría y literatura en la España de la transición: los renglones torcidos de Dios (1979). Rev Latinoam Psicopat Fund [Internet]. 2017 [citado em 2018 ago 15];20(1):142-64. doi: http://dx.doi. org/10.1590/1415-4714.2017v20n1p142.10

13. Damásio A. O erro de Descartes: emoção, razão e cérebro humano. Lisboa: Círculo de Leitores; 2011.

14. Damásio A. A estranha ordem das coisas. Lisboa: Círculo de Leitores; 2017.

15. Azagra-Caro J, Fernández-Mesa A, Robinson-Garcia N. 'Getting out of the closet': Scientific authorship of literary fiction and knowledge transfer. J technol transf [Internet]. 2018 [citado em 2018 ago 15]:1-30. doi: http://doi.org/10.1007/s10961-018-9672-6

16. Bladon H. Should psychiatrists write fiction? BJPsych Bull [Internet]. 2018 [citado em 2018 ago 15];42(2):77-80. doi: http://doi.org/10.1192/bjb.2017.5

17. Launer J. "Dying": what medicine can learn from fiction. Postgrad Med J [Internet]. 2018 [citado em 2018 ago 15];94(1107):69-70. doi: http://dx.doi.org/10.1136/postgradmedj-2017-135487

18. Sousa MS, Gallian D, Maciel R. Humanidades médicas no Reino Unido: uma tendência mundial em educação médica hoje. Rev Med (São Paulo) [Internet]. 2012 [citado em 2018 ago 15];91(3):163-73. doi: https://doi.org/10.11606/issn.1679-9836.v91i3p163-173 
19. Cavalcante LM, Leite AJM, Neves Filho AC, Sidrim PRP, Silva LS. Humanidades Médicas: para Quem e para Quê? Blucher Med Proc [Internet]. 2014 [citado em 2018 ago 15];1(2):357. doi: http://doi. org/10.5151/medpro-cihhs-10804

20. Ayres JSCM, Rios IC, Schraiber LB, Falcão MTC, Mota A. . Humanidades como Disciplina da Graduação em Medicina. Rev Bras Educ Med [Internet]. 2013 [citado em 2018 ago 15];37(3):455-63. doi: http:// dx.doi.org/10.1590/S0100-55022013000300019

21. Trajano VS, Carvalho ACC, Sawada ACMB, Jorge TCA. Ciência, arte e cultura na saúde. Rev Educ Artes Incl [Internet]. 2018 [citado em 2018 ago 15];14(2):134-51. Disponível em: http://www.revistas.udesc. br/index.php/arteinclusao/article/view/9853/pdf

22. Reginato $\mathrm{V}$, Gallian D, Marra S. Literature in the education of future scientists: a lesson of Frankenstein. Educ Pesqui [Internet]. 2018 [citado em 2018 ago 15];44:1-16. doi: http://dx.doi.org/10.1590/s1517$\underline{9702201610157176}$

23. Ferreira MA. Educação médica no século XXI: o desafio da integração da tecnologia e humanidades. Gaz Med [Internet]. 2016 [citado em 2018 ago 15];3(4):156-61. Disponível em: http://www.gazetamedica. pt/index.php/gazeta/article/view/42/64

24. Guardiola E, Baños JE. El papel de las humanidades médicas en la educación de los profesionales de la salud del siglo XXI. Rev Med Cine [Internet] 2017 [citado em 2018 ago 15];13(4):155-7. Disponível em: https://goo.gl/RXY7XY

25. Gomes T, Higa E, Passos A, Soares M, Otani M, Souto, B. A visão de estudantes de medicina e enfermagem sobre a integralidade na atenção primária à saúde. Rev Psicol Divers Saude. 2018;7(1):112-9. doi: http://dx.doi.org/10.17267/2317-3394rpds.v7i1.1898

26. Rios I. Humanidades Médicas como Campo de Conhecimento em Medicina. Rev Bras Educ Med [Internet]. 2016 [citado em 2018 ago 15];40(1):21-9. doi: http://dx.doi.org/10.1590/1981$\underline{52712015 v 40 n 1 e 01032015 .}$

27. Sanchez Gonzalez M. El humanismo y la enseñanza de las humanidades médicas. Educación Médica. [Internet]. 2017 [citado em 2018 ago 15];18(3):212-8. doi: https://doi.org/10.1016/j. edumed.2017.03.001 